Ann. Biol. anim. Bioch. Biophys., I97I, 11 (I), I03-II2.

\title{
COMPARAISON DES ASPECTS CYTOPHYSIOLOGIQUES DE LA MUQUEUSE DE POCHES FUNDIQUES ET DE L'ESTOMAC ENTIER CHEZ LE LAPIN
}

\author{
M. BEAUVILLE, P. RAYNAUD, G. PRADAL* et J. TUSQUES* \\ avec la collaboration technique de R. Bonnemarson et Josiane Valton
}

\author{
Institut de Physiologie animale, 84, grande rue Saint-Michel, \\ 31 - Toulouse \\ * Laboratoire d'Histologie et d'Embryologie, \\ Faculté mixte de Médecine et de Pharmacie, 1, rue Gaston-Veil, \\ 44 - Nantes
}

\section{RÉSUMÉ}

Le but de ce travail est de rechercher si la sécrétion d'une poche gastrique isolée est la même que celle de la région correspondante de l'estomac et si l'ultrastructure de la muqueuse de la poche est normale. L'étude a étó faite sur des Lapins munis de poches selon une technique originale.

Les résultats obtenus avec des animaux sacrifiés montrent que l'activité pepsinique et le pH du suc sécrété par la poche ainsi que la teneur en pepsinogène de la muqueuse ont des valeurs voisines de celles de la région correspondante de l'estomac.

A partir de prélèvements de muqueuse de poches effectués sur l'animal éveillé, nous avons pu nous rendre compte que la taille des cellules glandulaires est diminuée mais que l'ultrastructure demeure normale.

Nous avons pu également observer que l'apparition de pepsine dans le suc s'accompagne d'une vidange des grains de zymogène des cellules principales et, inversement, que lorsque les cellules sont abondamment garnies de grains, le suc contient très peu de pepsine. Par ailleurs, nous décelons au niveau du col l'existence de cellules mixtes contenant à la fois des canalicules intracellulaires et des grains de zymogène.

En conclusion, cette poche fonctionne comme la région correspondante de l'estomac, l'ultrastructure des cellules est normale, la sécrétion non pariétale est intacte ; seule la sécrétion pariétale semble légèrement diminuée.

\section{INTRODUCTION}

L'étude de la sécrétion gastrique est faite la plupart du temps sur des animaux munis d'une poche isolée afin que les aliments et les sécrétions salivaires ne viennent pas se mélanger à la sécrétion de l'estomac.

Mais la sécrétion d'une poche gastrique isolée est-elle la même que celle de la 
région correspondante de l'estomac? L'ultrastructure de la muqueuse de la poche est-elle identique à celle de l'estomac normal? Nous avons essayé de répondre à ces questions en utilisant le Lapin comme animal d'expérience.

\section{MATÉRIEI ET TECHNIQUES}

Les lapins utilisés sont de race commune, d'un poids moyen de $2,500 \mathrm{~kg}$; ils sont munis d'une poche gastrique isolée selon une technique précédemment décrite par deux d'entre nous (BEAUville et RAYNaUd, 1968). Cette poche est établie dans la région fundique de la grande courbure parce que la muqueuse de cette zone est la plus riche en pepsinogène (BEAUVILLE et RAYNAUD, 1963).

Lorsque la poche fonctionne convenablement, c'est-à-dire lorsque le suc sécrété redevient acide, les lapins sont placés dans une cage à contention après une période de conditionnement de quelques jours. Le suc gastrique est recueilli toutes les $3^{\circ}$ minutes dans les tubes d'un collecteur de fractions par l'intermédiaire d'un cathéter très souple abouché à la canule.

L'activité pepsinique du suc est évaluée sur une solution de blanc d'œuf coagulé en référence d'une solution de pepsine cristallisée (Nutr. Biochem. Corp.). Les résultats sont exprimés en microgrammes d'équivalent de pepsine par millilitre. Le pH est mesuré à l'aide d'un pHmètre Méthrom au centième.

Toutes les $2 \mathrm{~h}$ 30 le cathéter est débranché et un prélèvement de muqueuse est effectué à l'aide d'une pince à biopsies introduite directement par la canule jusque dans la muqueuse de la poche (fig. I). Cette partie de la manipulation est délicate; en effet, la muqueuse est très friable et il ne faut pas enfoncer la pince trop profondément dans la paroi de la poche pour ne pas léser la couche musculeuse. En général, nous ne faisons qu'une expérience par lapin et nous limitons a trois ou quatre le nombre des prélèvements.

Les fragments d'un millimètre cube environ sont fixés dans une solution tamponnée au véronal-acétate, d'acide osmique à $3 \mathrm{p}$. $100(\mathrm{pH}: 7,7)$ pendant deux heures, puis déshydratés et inclus dans l'Epon 812. Des coupes d'environ un micron sont faites à l'ultramicrotome Reichert pour la microscopie optique ; celles-si sont colorées au bleu de toluidine, examinées et photographiées avec un microscope Leitz. Des coupes de 500 à 600 Angströms sont faites avec le même ultramicrotome pour la microscopie électronique, puis sont doublement colorées à l'acétate d'uranyle et au citrate de plomb, enfin examinées avec un microscope Hitachi HU i I C. S.

Nous avons en outre sacrifié sept lapins en vue d'établir une comparaison à un instant donné, entre les constituants du suc sécrété par la poche et ceux du matériel stomacal dans la région voisine. Pour faciliter la lecture des résultats, nous avons arbitrairement divisé l'estomac en six zones le long de la grande courbure, la zone I correspond au cul-de-sac fundique, la zone 6 à l'antre pylorique, la poche étant située au niveau de la zone 2.

\section{RÉSULTATS}

\section{Généralités}

Les poches gastriques isolées chez le Lapin sécrètent de façon continue un suc acide (BEAUVILLE et RAYNAUd, I964; Limbosch et al., I966). Le débit varie d'un lapin à l'autre étant donné que toutes les poches n'ont pas la même surface; en général, il se situe aux environs de trois millilitres par heure. Alors que le débit et 1'acidité sont à peu près constants, la concentration en pepsine subit des variations considérables (BEAUVILLE et coll., I966). Il faut noter tout de même que la sécrétion des poches ne devient acide que quelques jours après l'opération et que parfois, sans doute à cause d'une préparation défectueuse, certaines poches continuent à sécréter un suc peu abondant et qui reste neutre. 
En microscopie optique et en microscopie électronique, on retrouve dans la muqueuse de la poche isolée toutes les cellules que l'on rencontre habituellement dans la muqueuse fundique. Cependant, alors que l'épithélium de surface semble tout à fait normal, l'épithélium glandulaire présente une diminution de l'épaisseur des glandes liée à une réduction de taille des cellules bordantes et des cellules principales. De plus la lamina propria présente un aspect œématié.

\section{Cellules bordantes}

En microscopie optique, sur des coupes " semi-fines " colorées au bleu de toluidine, il apparaît que les cellules bordantes de la poche isolée sont de plus petite taille que celles de la muqueuse fundique normale. Ces cellules à contours nets se prêtent aisément à des mensurations. Nous avons dessiné à la chambre claire des cellules bordantes avec leur noyau; un grossissement de I 300 fois mesuré à l'aide d'un micromètre objectif a été utilisé.

Nous avons mesuré $\mathrm{I}$ ooo cellules et $\mathrm{I}$ ooo noyaux répartis entre les deux lots d'animaux, six animaux témoins et six poches isolées. Les résultats de ces mesures exprimés sur la figure A montrent que les cellules bordantes des animaux témoins

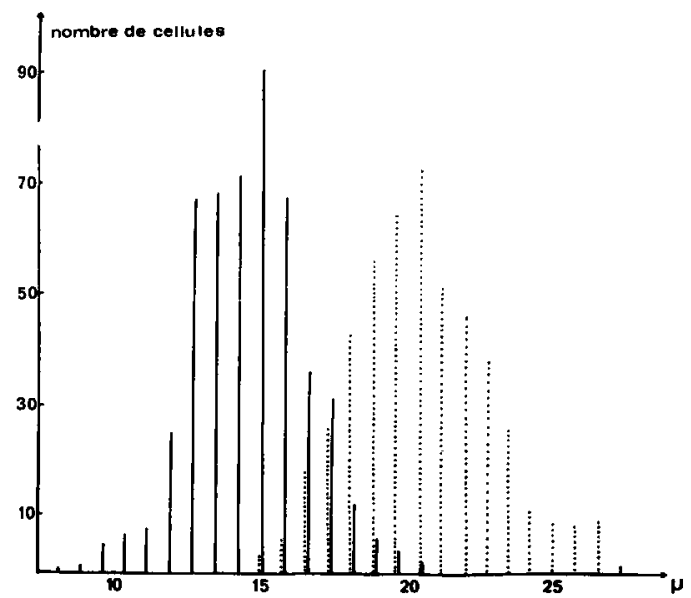

Fig. A. - Comparaison du diamètre des cellules bordantes de la poche (traits pleins) et de l'estomac (traits pointillés). Le diamètre, en abscisses, est exprimé en microns

sont plus grandes que celles des animaux munis d'une poche isolée. Par contre, on n'observe pas de différence très nette pour les noyaux (fig. B).

Les résultats moyens obtenus sont les suivants :

- pour les cellules:

chez les témoins

chez les animaux d'expérience

$d=20,2 \pm 2,3$ microns

- pour les noyaux :

$d=\mathrm{I} 4,3 \pm 0,5$ microns

chez les témoins

$d=5,8 \pm \mathrm{I}, 0$ microns

chez les animaux d'expérience

$d=4,6 \pm \mathrm{I}, 0$ microns 
On constate en définitive que les cellules bordantes de la poche sont plus petites que celles de l'estomac, tandis que la diminution de taille des noyaux n'est pas significative. Il en résulte que seul le cytoplasme des cellules bordantes subit une modification ; dans ces conditions, il est évident que le rapport nucléoplasmique est plus élevé dans les cellules bordantes de la poche que dans celles de l'estomac.

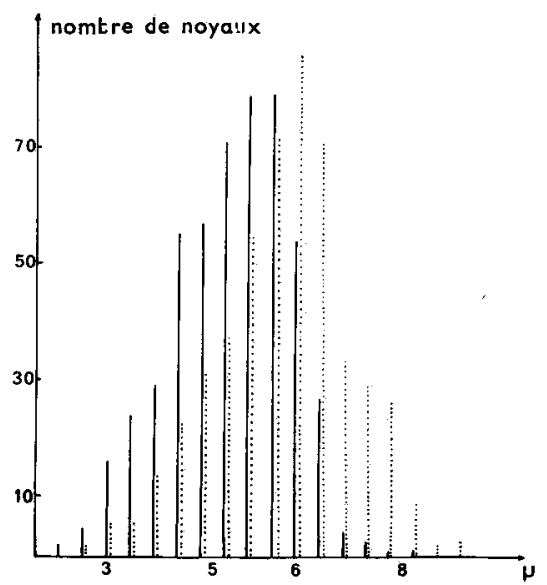

FIg. B. - Comparaison du diamètre des noyaux des cellules bordantes de la poche (traits pleins) et de l'estomac (traits pointillés)

La microscopie électronique nous a permis de constater que dans un prélèvement donné, la physionomie des cellules bordantes est assez homogène du point de vue de leur état sécrétoire; seul un nombre restreint de cellules dans l'ensemble de cette population se trouve dans un état sécrétoire différent. Les critères cytologiques permettant d'apprécier les différences dans l'état sécrétoire de ces cellules sont constitués par le degré d'ouverture des microcanalicules intracellulaires et la densité des microvillosités qui bordent ceux-ci. Nous donnons un exemple de ce phénomène dans lequel la majorité des cellules bordantes possède des microcanalicules entrouverts avec de nombreuses microvillosités (fig. 2) ; cependant un petit nombre de cellules, disséminées d'ailleurs, présentent des microcanalicules très largement ouverts bordés seulement de quelques rares microvillosités.

Les habituels organites intracytoplasmiques sont normalement représentés. En effet, le réticulum endoplasmique apparaît sous forme de vésicules lisses, quelquefois au contact des microcanalicules. Les polysomes, formations permanentes de la cellule bordante, sont ici très bien représentés (fig. 3 et 4). Les mitochondries ont un aspect banal ; elles comportent des crêtes serrées (fig. 4), toutefois il faut signaler que certaines de celles-ci sont altérées et présentent en leur sein des figures myéliniques localisées (fig. 3). On observe en outre la présence assez régulière de flaques lipidiques en général au voisinage des mitochondries (fig. 4).

Enfin nous avons rencontré, au niveau du col, un írès pesit nombre de cellules bordantes comportant des grains analogues aux grains de zymogène (fig. 5) par leur taille d'abord (I à 2 microns) et par la présence localement visitle d'une membrane tristratifiée. Il faut noter que ces grains apparaissent peu denses aux électrons ; sans doute leur contenu a-t-il diffusé dans l'hyaloplasme. Le noyau est en outre très 
encoché à l'inverse des cellules bordantes précédemment décrites qui ont un noyau vésiculeux.

L'essentiel des variations morphologiques et ultrastructurales des cellules bordantes porte donc en définitive sur une diminution du cytoplasme et une réduction proportionnelle $\mathrm{d} u$ nombre de mitochondries. Les données physiologiques montrent pourtant que le suc sécrété par la poche est toujours acide, le $\mathrm{pH}$ se situant en général entre 0,9 et $\mathrm{I}, 5$ (tabl. I) et il est relativement stable pendant la durée de l'expérience ; de plus ce $\mathrm{pH}$ est de l'ordre de celui que l'on rencontre dans l'estomac entier.

\section{TABLEAU I}

Données cytophysiologiques sur le fonctionnement de la poche isolée

Le $\mathrm{pH}$ est mesuré pendant la demi-heure qui précède la biopsie

La pepsine est exprimée en microgramme par millilitre

++ signifie : cellule principale abondamment garnie de grains de zymogène ; + : avec quelques grains; 0 : sans grains.

\begin{tabular}{|c|c|c|c|c|c|c|c|c|c|c|c|c|}
\hline \multirow[b]{2}{*}{ Lapin } & \multicolumn{3}{|c|}{$1^{\text {er }}$ prélèvement } & \multicolumn{3}{|c|}{$2^{\mathrm{e}}$ prélèvement } & \multicolumn{3}{|c|}{$3^{\mathbf{e}}$ prélèvement } & \multicolumn{3}{|c|}{$4^{\mathrm{e}}$ prélèvement } \\
\hline & $\mathrm{pH}$ & Pepsine & Grains & $\mathrm{pH}$ & Pepsine & Grains & $\mathrm{pH}$ & Pepsine & Grains & $\mathrm{pH}$ & Pepsine & Grains \\
\hline $\mathrm{E}$ & 1,38 & 15 & ++ & 1,50 & 5 & ++ & 1,31 & 5 & ++ & 1,32 & 40 & ++ \\
\hline A & 1,26 & 5 & ++ & 1,14 & 5 & ++ & 1,12 & 10 & ++ & 1,20 & 50 & ++ \\
\hline $\mathrm{F}$ & 1,30 & 10 & ++ & 1,34 & 10 & ++ & 1,31 & 30 & ++ & 1,45 & 55 & ++ \\
\hline $\mathrm{J}$ & 1,04 & 10 & & 1,03 & 40 & + & 0,99 & 85 & ++ & & & \\
\hline $\mathbf{H}$ & 1,34 & 10 & + & 1,22 & 110 & ++ & 1,19 & 120 & + & & & \\
\hline I & 1,12 & 30 & ++ & 1,10 & 110 & ++ & 1,10 & 120 & + & & & \\
\hline B & 1,36 & 80 & ++ & 1,52 & 100 & ++ & 1,40 & 150 & ++ & & & \\
\hline G & 1,31 & 105 & ++ & 1,22 & 230 & ++ & 1,22 & 375 & + & 1,26 & 320 & + \\
\hline $\mathrm{C}$ & 1,22 & 30 & + & 1,21 & 65 & & 1,32 & 1100 & $\mathrm{O}$ & & & \\
\hline $\mathrm{E}$ & 1,20 & 950 & 0 & 1,20 & 700 & 0 & 1,38 & 700 & 0 & 1,25 & 650 & 0 \\
\hline D & 0,94 & 5 & 0 & 0,98 & 765 & + & 0,97 & 1125 & 0 & 0,98 & 1650 & 0 \\
\hline
\end{tabular}

\section{Cellules principales}

L'étude comparée du contenu des cellules principales et de la teneur en pepsine du suc sécrété montre que, d'une façon générale, l'apparition de pepsine dans le suc s'accompagne d'une perte, en tous cas d'une absence de grains, dans les cellules principales (tabl. I). La présence ou l'absence de grains de zymogènes est décelable en microscopie optique comme le montrent les figures 6 et 7 . On remarque également que les cellules de la poche isolée sont de taille plus petite que les cellules de l'estomac normal. Il ne nous a pas été possible d'effectuer des mensurations étant donné le délomor- 
phisme de ce type cellulaire, toutefois la diminution de taille semble être du même ordre de grandeur que celle des cellules bordantes.

Après sacrifice de lapins munis de poche, nous avons pu nous rendre compte que, malgré les variations individuelles très importantes, la teneur en pepsine du suc sécrété par la poche est voisine de celle observée dans la région correspondante de l'estomac (tab1. 2).

TABLEAU 2

Activité pepsinique dans le suc sécrété par la poche et dans le matériel stomacal chez les animaux sacrifiés

\begin{tabular}{|c|c|c|c|c|c|c|c|}
\hline \multirow{3}{*}{ Poche } & \multicolumn{7}{|c|}{ Activité pepsinique en microgramme/ml } \\
\hline & \multicolumn{6}{|c|}{ Matériel stomacal } & \multirow{2}{*}{$\begin{array}{l}\text { Nombre de } \\
\text { jours après } \\
\text { opération }\end{array}$} \\
\hline & 1 & 2 & 3 & 4 & 5 & 6 & \\
\hline 40 & 0 & 0 & 215 & 430 & 475 & 285 & 27 \\
\hline 180 & 0 & 40 & 430 & 720 & 480 & 460 & 36 \\
\hline 180 & 0 & 230 & 520 & 580 & 600 & 480 & 45 \\
\hline 480 & 310 & 540 & 1200 & 1100 & 1200 & 625 & 35 \\
\hline 820 & 110 & 410 & 380 & 480 & 440 & 300 & 10 \\
\hline 880 & 760 & 850 & 640 & 1040 & 880 & 550 & 23 \\
\hline 3700 & 1200 & 1310 & 1420 & 1510 & 1000 & 750 & 21 \\
\hline
\end{tabular}

Comme chez l'animal normal, les séquences d'images observées montrent qu'à un moment donné, les cellules principales d'un prélèvement muqueux se trouvent toutes au même état sécrétoire. La figure ro qui correspond au premier prélèvement du lapin $G$ (tabl. I) montre une cellule renfermant de nombreux grains de zymogène immatures car leur contenu finement granuleux est encore peu dense aux électrors. Ces grains occupent une position supranucléaire et ne sont pas, à ce stade, circorscrits par une membrane limitante. La figure 8 correspondant au dernier prélèvement du lapin E (bas du tableau I) met en évidence des cellules principales entièrement dégranulées ; la totalité de 1'hyaloplasme étant occupée par de l'ergastoplasme. On rencontre également des cas intermédiaires; les cellules principales peuvent par exemple ne renfermer que quelques grains résiduels, généralement très dense aux électrons ; ces grains sont alors circonscrits par une membrane limitante tristratifée (fig. I2). Nous notons également la netteté particulière des membranes basales (fig. 9) (ce fait est d'ailleurs commun aux autres types cellulaires). Signalons enfin la présence inhabituelle pour ce type de cellule, de gouttelettes de lipides au sein de l'ergastoplasme (fig. II).

\section{Autres types cellulaires}

En microscopie optique on constate que l'épi ihélium superf.ciel form.é de cellules muqueuses a un aspect tout à fait banal ; on y observe des grains de mucus apicaux fortement colorables par le bleu de toluidine (fig. I4). Ces cellules ont une taille et une morphologie identiques à celles de l'épithélium stomacal normal (fig. 13).

En microscopie électronique également ces cellules sont très comparables à 
celles de la muqueuse normale. L'apex des cellules est occupé par un grand nombre de grains denses aux électrons (fig. I5). La membrane tristratifiée qui limite ces grains est ici visible (fig. I6) alors qu'elle ne l'est généralement pas chez les animaux témoins en raison précisément de l'extrême densité de leur contenu. Au voisinage de lappareil de Golgi on observe des grains en voie de formation (fig. I8) ; les mitochondries ont l'aspect habituel de ce type cellulaire c'est-à-dire allongées et comportant des crêtes espacées. Ces cellules présentent donc un aspect normal et les organites habituels sont particulièrement bien préservés. Il faut noter cependant la fréquence inhabituelle de corps multivésiculaires (fig. I6) et également de lysosomes de taille souvent volumineuse dans un assez grand nombre de cellules (fig. I7).

Les cellules du col peu nombreuses et groupées, comme dans la muqueuse fundique normale, présentent une ultrastructure tout à fait typique. Les grains de mucus, apicaux, ont une membrane limitante peu visible (fig. I9). Le contenu de ces grains est peu dense aux électrons. L'appareil de Golgi apparaît sous forme de saccules et de vésicules.

Enfin, nous notons que les cellules argyrophiles sont peu nombreuses et disséminées. Les grains peuvent être circonscrits par une membrane limitante (fig. 20). On rencontre dans l'hyaloplasme, mais rarement, des plages ou gouttelettes lipidiques. Nous avons rencontré en outre l'inclusion intranucléaire d'allure filamenteuse (Tusques et PradaI, I968 a).

\section{DISCUSSION}

Le simple examen en microscopie optique suggère que la muqueuse de la poche fonctionne normalement. En effet, nous retrouvons toutes les cellules qui ont été décrites dans la muqueuse de l'estomac normal.

Néanmoins, la réduction de la taille des cellules de l'épithélium glandulaire s'accompagne sans doute d'une diminution de la quantité de la sécrétion de ces cellules. Par ailleurs, l'aspect œdématié de la lamina propria doit certainement traduire une difficulté de la circulation de retour.

Un examen plus approfondi met en évidence, comme dans l'estomac entier, des quantités variables de grains de zymogène dans les cellules principales, des grains de sécrétion à différents stades dans les cellules argyrophiles et des canalicules plus ou moins ouverts dans les cellules bordantes.

L'examen en microscopie électronique des cellules de la muqueuse de la poche permet en outre, de se rendre compte que les organites intracellulaires classiquement décrits sont présents. Il faut signaler tout de même quelques légères figures d'altération; en effet, dans les cellules bordantes certaines mitochondries peuvent présenter des figures myéliniques localisées; dans les cellules muqueuses superficielles se rencontrent des corps mulivivésiculaires et des lysosomes.

La prés ənce de flaques lipidiques dans les cellules bordantes et principales n'est pas exceptionnelle mais leur nombre est bien plus élevé que dans la muqueuse normale. Pareille constatation a été rapportée par HAYWARD chez le Lapin nouveau-né (I967). Les caractères ultrastructuraux des rares cellules bordantes contenant des grains analogues aux grains de zymogène, suggèrent qu'il pourrait s'agir de cellules en voie de différenciation étant donné leur situation au voisinage du col dont on sait 
qu'il est la zone de renouvellement et de différenciation cellulaire (LEBLOND et WALKER, I956 ; HUNT, I958 ; MYRHE, I960 ; HUNT et HUNT, I962). C'est, à notre connaissance, le seul type de cellule mixte décrite chez un Mammifère, alors que ce type cellulaire est la règle chez les Poissons, les Reptiles (ITO, I 967), les Batraciens (NORRIs, I959; SEDAR, I96I) et chez les Oiseaux (TONER, I963).

Une très forte similitude existe entre les cellules de la poche et celles de l'estomac entier pour ce qui concerne à la fois l'aspect cytologique et le fonctionnement. L'ultrastructure des cellules bordantes (Tusques et PRADAL I968 $b$ ) montre en effet que dans un prélèvement donné, l'état sécrétoire est le même pour la plupart des cellules, tandis qu'un petit nombre de celles-ci semble au repos. Au point de vue physiologique (ALEXANDER et ChowdHURY, I958; SEKINE, I966) la sécrétion des poches est aussi toujours acide, le $\mathrm{pH}$ est très voisin de $\mathrm{I}$.

De plus, le suc sécrété contient des teneurs en pepsine très variables et nous observons, comme pour 1'estomac entier, des variations individuelles et des variations dans le temps. En ce qui concerne l'ultrastructure des cellules principales nous remarquons aussi (PRADAI et TusguEs, I968) que dans un prélèvement donné toutes les cellules se trouvent dans le même état sécrétoire et nous observons en outre qu'il se produit une décharge massive de la totalité du zymogène qui va de pair avec l'apparition d'une activité pepsinique très importante dans le suc. Inversement, l'accumulation de grains de zymogène dans les cellules correspond à une activité pepsinique très faible.

En dehors des observations concernant les cellules principales ou bordantes, il nous paraît intéressant de rapporter quelques faits concernant les autres types de cellules.

Les cellules muqueuses de surface rappellent assez exactement celles de l'estomac entier. Toutefois, les grains de mucus situés à l'apex de ces cellules sont moins denses aux électrons que pour la muqueuse normale (KUROSOMI et coll., I958 ; HELANDER, I962; ITo, I963; RUBIN et coll., I968). Il faut ajouter que les corps multivésiculaires et les lysosomes sont plus nombreux et que ces derniers ont le plus souvent une taille volumineuse.

Les cellules muqueuses du col observées au niveau de la muqueuse de la poche présentent exactement les mêmes caractéristiques que leurs homologues de l'estomac ; on y retrouve notamment des grains dont la densité est faible aux électrons ce qui est typique de ce genre de cellule (HELANDER, I962 ; ITO et WiNCHESTER, I963 ; LILLIBRIDGE, Ig64).

Nous retrouvons pour les cellules argyrophiles les caractéristiques ultrastructurales décrites pour l'estomac entier (RATZENHOFER et coll, I965; RATZENHOFER, I966; Tusques et PRADAI, I968 a). Ces auteurs attribuent à ces cellules une autonomie fonctionnelle; cette hypothèse pourrait être vérifiée sur des poches isolées.

\section{CONCLUSIONS}

Du point de vue qualitatif la muqueuse de la poche seproduit très certainement tous les mécanismes qui aboutissent à la fabrication d'un suc gastrique normal puisque nous retrouvons tous les types cellulaires et leurs organites ainsi que les variations morphologiques déjà signalées sur l'estomac entier. 
Nous relevons dans la poche les aspects cytologiques qui indiquent que, comme dans l'estomac entier, la production d'acide est continue et qu'elle résulte d'un véritable " tonus sécrétoire " des cellules bordantes. Les observations que nous faisons sur les cellules principales rendent compte des variations très importantes de l'activité pepsinique du suc.

Du point de vue quantitatif nous avons naturellement quelques restrictions à apporter car la diminution de taille des cellules bordantes et principales, bien que n'affectant pas le noyau, entraîne certainement une réduction de la quantité d'acide et de zymogène produits. Par contre, la taille des' cellules muqueuses demeure inchangée ce qui laisse supposer que la sécrétion de ces cellules n'est pas affectée.

Reçu pour publication en novembre 1970.

\section{SUMMARY}

\section{CYTOPHYSIOLOGICAL, COMPARISON BETWEEN ISOLATED FUNDIC POUCH AND PARENT STOMACH MUCOSA IN THE RABBIT}

The current study was undertaken in order to determine whether the secretion of a gastric pouch isolated by means of an original technique is similar to that of the correspondings tomach area and whether the fine structure of the pouch mucosa is normal.

The results for sacrificed rabbits show that peptic activity, pouch juice $\mathrm{pH}$ and pepsinogen rate of the mucosa amounted to values similar to those of the corresponding stomach area.

Sections of gastric mucosa from the wake animal entitled us to observe that glandular cells were smaller in size, though their fine structure was normal.

The occurrence of pepsin in the gastric juice was found to be paralleled by a release of zymogen granules from the chief cells ; and, conversely, the number of zymogen granules is the higher when the pepsine contents of the juice is the lower. Moreover, dual cells showing both intracellular caniculi and zymogen granules were noticed in the neck region.

The pouch therefore appears functionally similar to its corresponding stomach area; its fine structure and non-parietal secretion appear normal. Only the parietal secretion rate seems feebly decreased.

\section{RÉFÉRENCES BIBLIOGRAPHIQUES}

Alexander F., Chowdhury A. K., I958. Digestion in the rabbi's stomach. Brit. J. Nutr., 12, $65-73$.

Beauville M., Raynaud P., 1963. Recherches sur l'activité peptique du contenu stomacal du Lapin pendant le nycthémère. J. Physiol., Paris, 55, 625-629.

Beauville M., Raynaud P., r964. Existence d'une sécrétion gastrique continue chez le Lapin. $J$. Physiol., Paris, 56, 287-288.

Beauville M., Pradal G., Raynaud P., I966. Influence du jeûne sur les constituants du suc gastrique chez le Lapin. C. R. Soc. Biol., 160, 404-408.

Beauville M., Raynaud P., I968. Nouvelle technique de confection d'une poche gastrique isolée chez le Lapin. C. R. Acad. Sci., Paris, 267, II53-I I56.

HaYward A. F., 1967. The ultrastructure of developing gastric parietal cells in the foetal rabbit. $J$. Anat., 101, 69-8I.

Helander H. F., 1962. Ultrastructure of fundus glands of the mouse gastric mucosa. J. Ultrastruct. Res., Suppl., 4, I-123.

Hunt T. E., 1958. Regeneration of the gastric mucosa in the rat. Anat. Record., 131, I93-212. 
Hunt T. E., Hunt E. A., I962. Radioautographic study of proliferation in the stomach of the rat using thymidine $\mathrm{H}^{3}$ and compound 48/80. Anat. Record, 142, 505-517.

Ito S., Winchester R. J., I963. The fine structure of the gastric mucosa in the bat J. Cell. Biol., 16, 54I-577.

Iтo S., r967. Anatomic structure of the gastric mucosa. In Charles F. Code : Alimentary Canal, vol. II 705-741. Waverly Press Baltimore.

Kurosumi K., Shibasaki S., Uchida G., Tanaka Y., 1958. Electron microscope studies on the gastric mucosa of normal rats. Arch. Histol. Japan., 15, 587-624.

Leblond C. P., WAIKer B. E., 1956. Renewal of cell populations. Physiol. Rev., 36, 255-276.

LiLlibridge C. B., r964. The fine structure of normal human gastric mucosa. Gastroenterology, 47, 269-29r.

Limbosch J. M., Wylute J. H., Fletcher T. L., Harkins H. N., Nyhus L. M., Ig66. Initial observation on gastric physiology in the rabbit. Surgical Forum, 17, 3II-3I3.

MYRHE E., 1960. Regeneration of the fundic micosa. V. An autoradiographic study on the effect of cortisone. Arch. Pathol., 70, 476-485.

NoRRIs J. L., 1959. Normal histology of the oesophogeal and gastric mucosae of the frog, Rana pipiens, J. Exptl. Zool., 141, I55-I74.

Pradal G., Tusques J., I968. Mise en évidence en microscopie électronique d'un cycle sécrétoire des cellules principales de l'estomac du Lapin dans le cadre de la sécrétion continue. J. Physiol, Paris, 69, $291-292$.

Ratzenhoffer M, Leb D., I965. Über die feinstruktur der argentaffinen und der Anderen Erscheinungsformen der "Hellen Zellen "feyrter's im kaniwchen-magen. Z. Zellforsch, 67, iI3-150.

Ratzenhofer M., r966. Zur biologie der endokrinen zellen (der Helle-Zelle Organs Feyrter) im verdauugstrakt (Nach Untersuchunger am Kaninchenmagen). Klin. Wscht. Dtsch., 44, rog-I 15.

Ritchie W. P., Delaney J. P., Barkilat A., Molina E., Wangensteen O. H., I966. Histological differencies between Heidenhain pouches and their parent stomachs. Surgery, 60, 1224-r228.

Rubin W., Ross I. L., Sleinsenger M. H., Jeffries G. H., 1968. The normal human gastric epithelia. A fine structural study. Lab. Invest., 19, 598-626.

SEDAR A. W., I96r. Electron microscopy of the oxyntic cell in the gastric glands of the bullfrog (Rana catesbiana). I. The non-acid secreting gastric mucosa. J. Biophys. Biochem. Cytol., 9, I-I8.

SEKINE T., I966. Effect of antrectomy or vagotomy on the sensitive of parietal cells to histamine admi nistered to the submucosa in the fundic gland area of the rabbit stomach. Tohoku J. Exp. Med., 88, 327-340.

ToNer P. G., I963. The fine structure of resting and active cells in the submucosal glands of the fowl proventriculus. J. Anat, 97, 575-583.

Tusques J., Pradal, I968 a. Cycle canaliculaire dans les a cellules bordantes de l'estomac du Lapin en microscopie électronique. 53e réunion Assoc. Anat., 7-I I avril (sous presse).

Tusques J., Pradal G., I968 b. Inclusion d'aspect filamenteux dans le noyau des cellules argyrophiles de la muqueuse gastrique du Lapin, mise en évidence en microscopie électronique. $C$. $R$. Acad. Sci., Paris, 267, I 738-I74I.

\section{PLANCHE, I}

FIG. I. - Coupe longitudinale d'un estomac muni d'une poche isolée (P. I. ). La pince introduite par la canule (cn.) permet d'effectuer les prélèvements. $\mathrm{Ca}$, : cardia ; Py. : pylore.

FIG. 2. - Cellule bordante comportant des microcanalicules presque fermés (flèches) bordés de nombreuses microvillosités. L. G. : lumière glandulaire. x 9000 . 

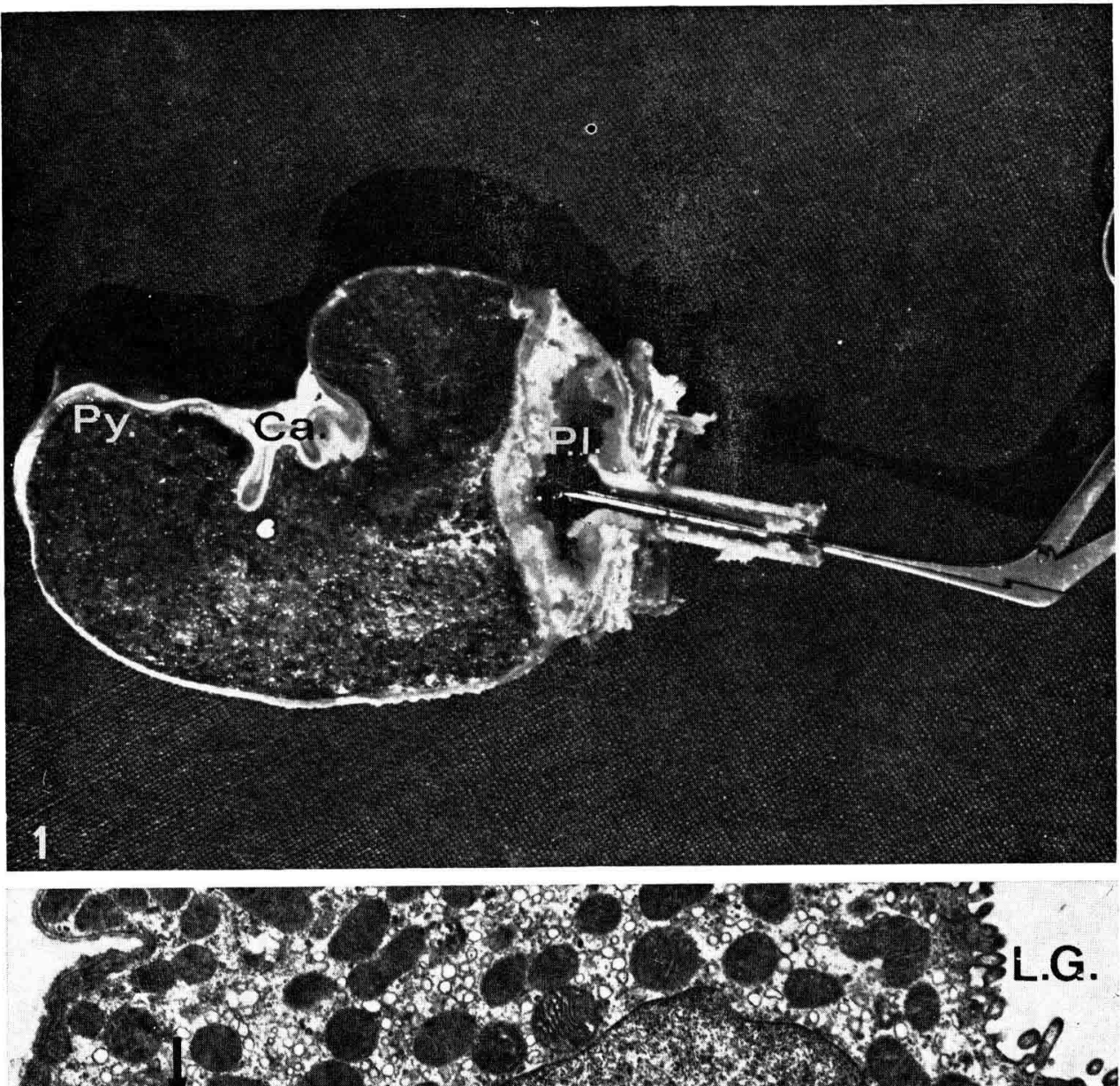

W.

V. 1.

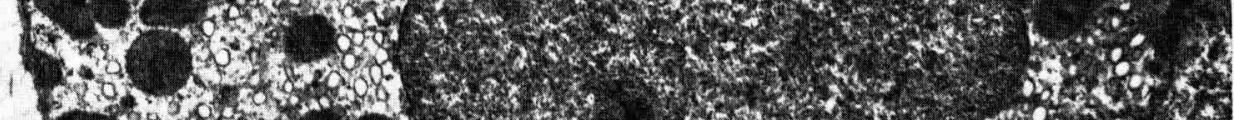

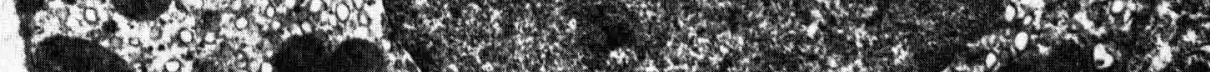
1.m.

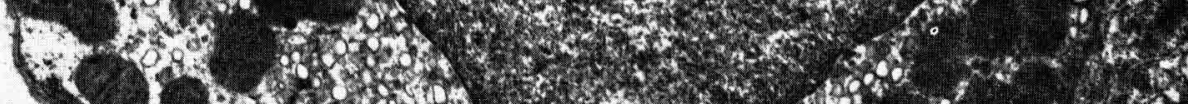

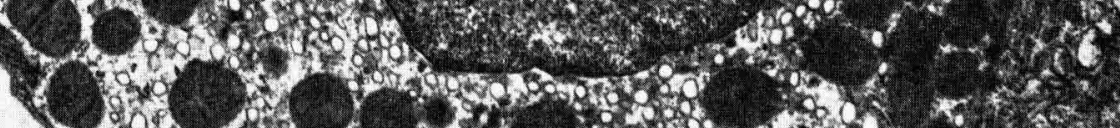

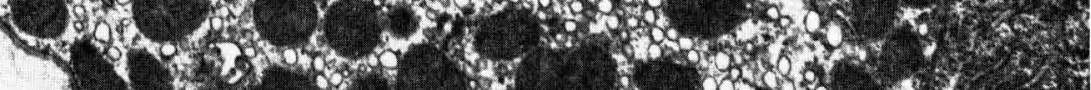

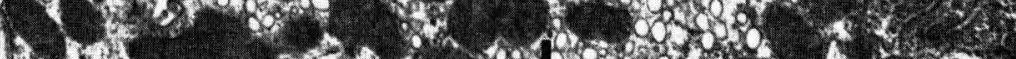

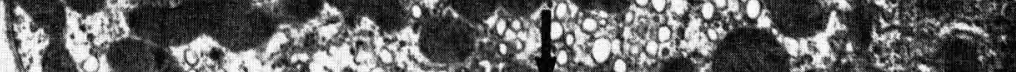

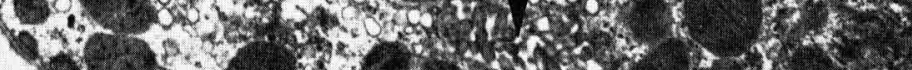




\section{PLANCHE II}

FIG. 3.

Mitochondrie $(\mathrm{m}$.$) de cellule bordante présentant une image$ myélinique. $\times 42000$.

FIG, 4.

Gouttelette lipidique (G. L.) dans une cellule bordante. On voit également des mitochondries (m.) d'allure banale et un microcanalicule entr'ouvert (m. c.) $\times 20000$.

Fig. 5.

Cellule mixte présentant, comme les cellules bordantes, des canalicules bordés de microvillosités (grandes flèches), des vésicules lisses (petites flèches), des polysomes et des mitochondries. On note aussi la présence de très nombreux granules clairs pouvant s'apparenter soit aux grains de zymogène, soit aux grains de mucus. Le noyau est très encoché à l'inverse des cellules bordantes typiques. $\times 12000$.

\section{FIG. 6 .}

Cellules principales (C. P.) abondamment garnies de grains de zymogène et cellules bordantes (C. B.) comprenant de très nombreuses mitochondries. $\times 2500$.

\section{FIG. 7.}

Cellules principales (C. P.) dégranulées. L. G. : lumière glandulaire. C. B. : cellules bordantes. $\times 2500$. 

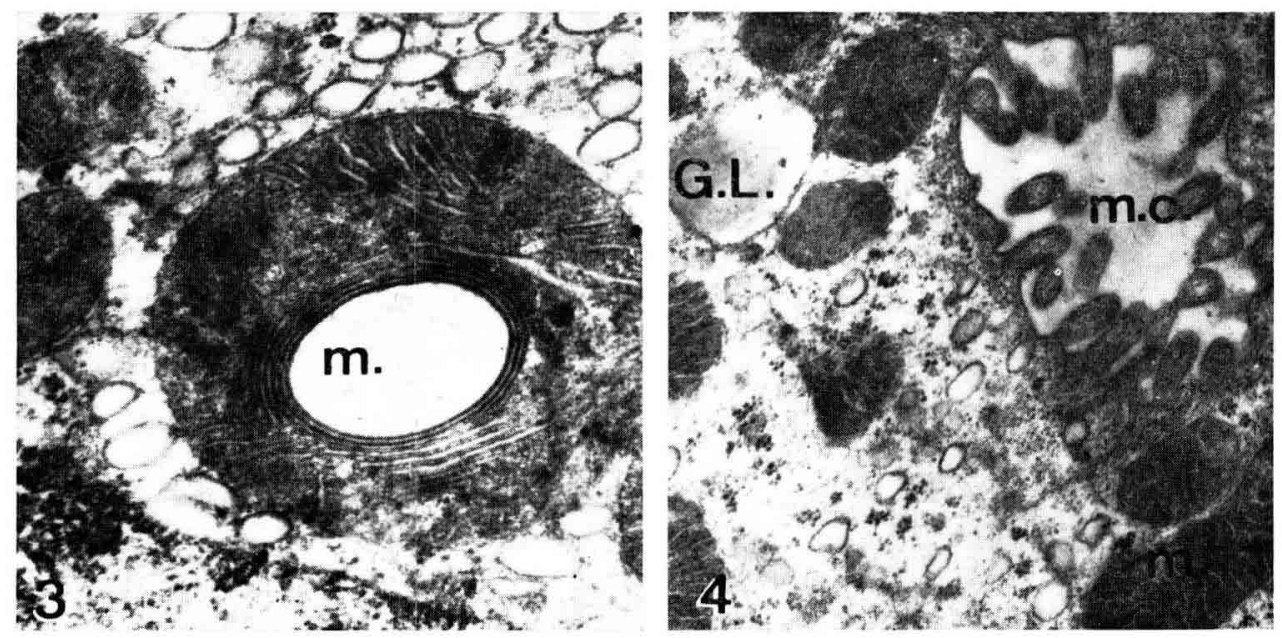

Q

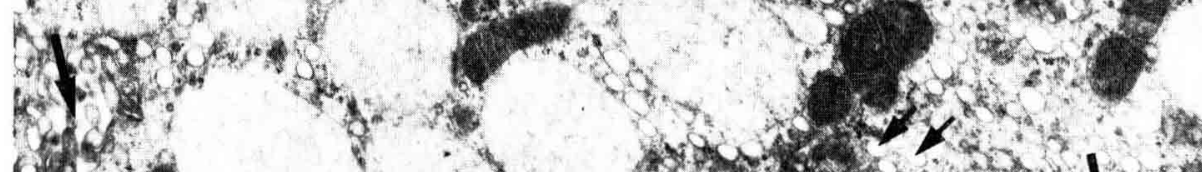

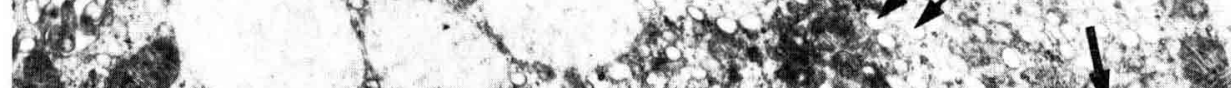

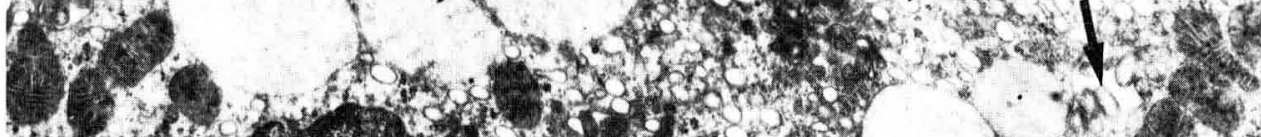

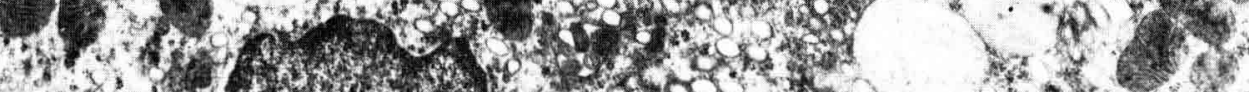

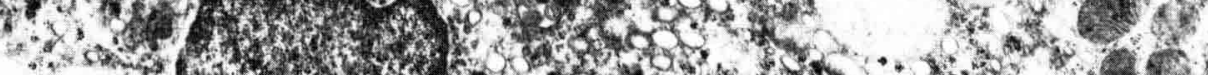

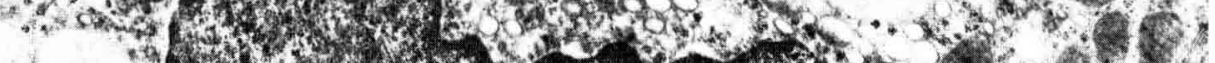

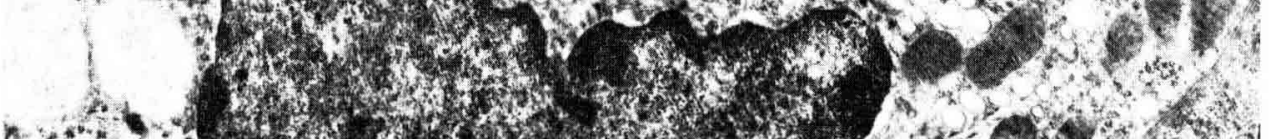

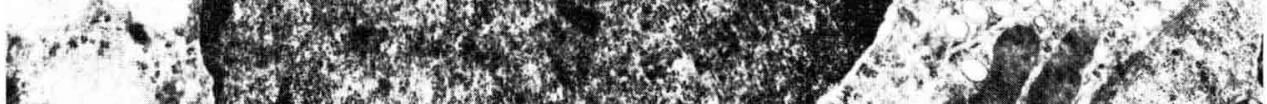

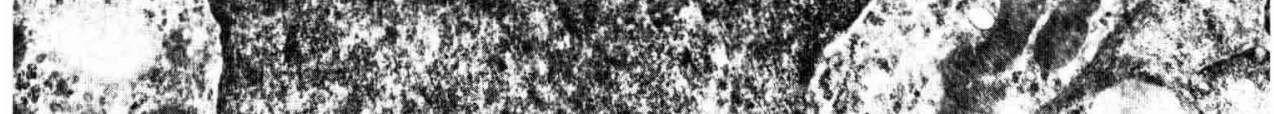

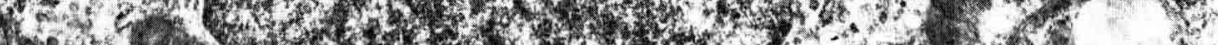
W.

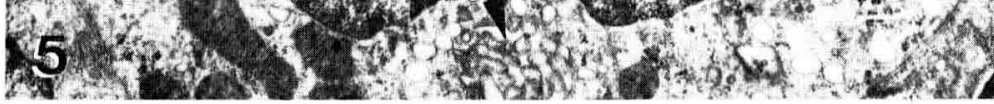
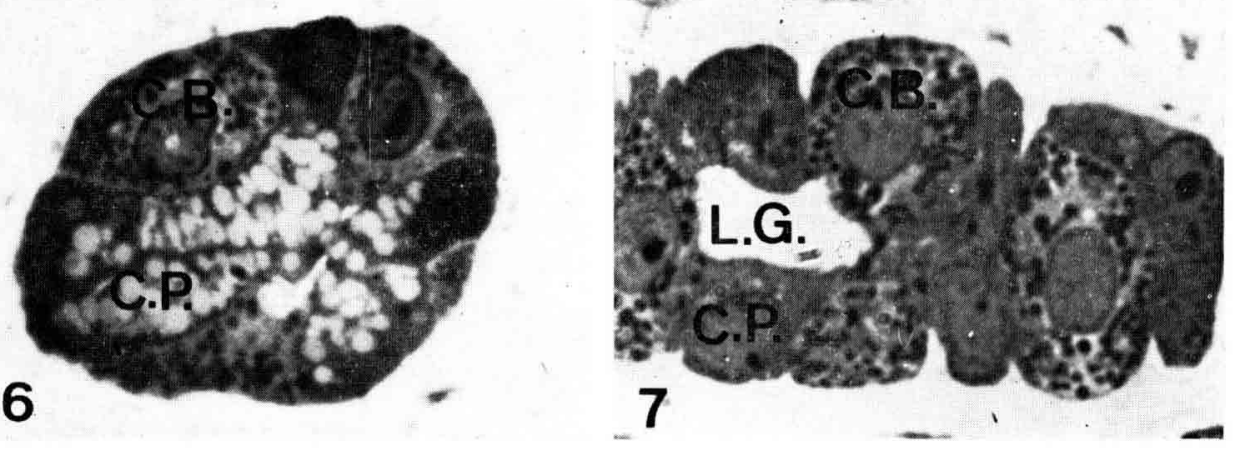


\section{PLANCHE III}

FIG. 8.

Cellules principales dégranulées (cp) ; L. G. : lumière glandulaire ; Portion de cellule bordante (cb). $\times 7500$.

FIG. 9.

Membrane plasmique basale et membrane basale. $\times 45000$.

FIG. 10.

Cellule principale contenant un grand nombre de granules de zymogène (Z.) supranucléaires. Les grains ne sont pas limités par une membrane tandis que l'ergastoplasme vient à leur contact (flèches). L. G. : lumière glandulaire. N. : noyau. × 12000 .

FIG. 11.

Gouttelette lipidique (G. L.) au sein de l'ergastoplasme (e.) d'une cellule principale. $\times 25000$.

FIG. 12.

Grains de zymogène mâtures (Z.) denses aux électrons ; ils sont circonscrits par une membrane tristratifiée (flèches). $\times \mathbf{4 0} 000$. 


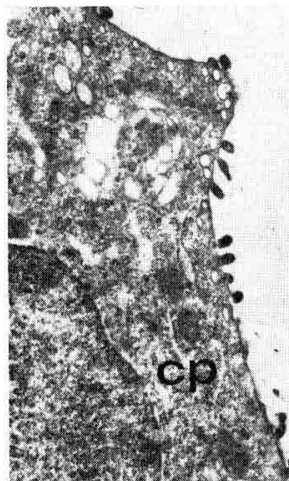

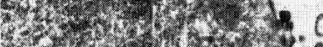

X

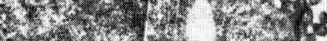

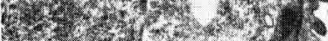

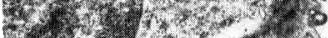

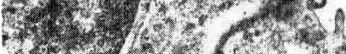

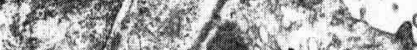

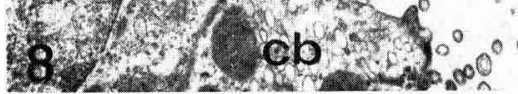

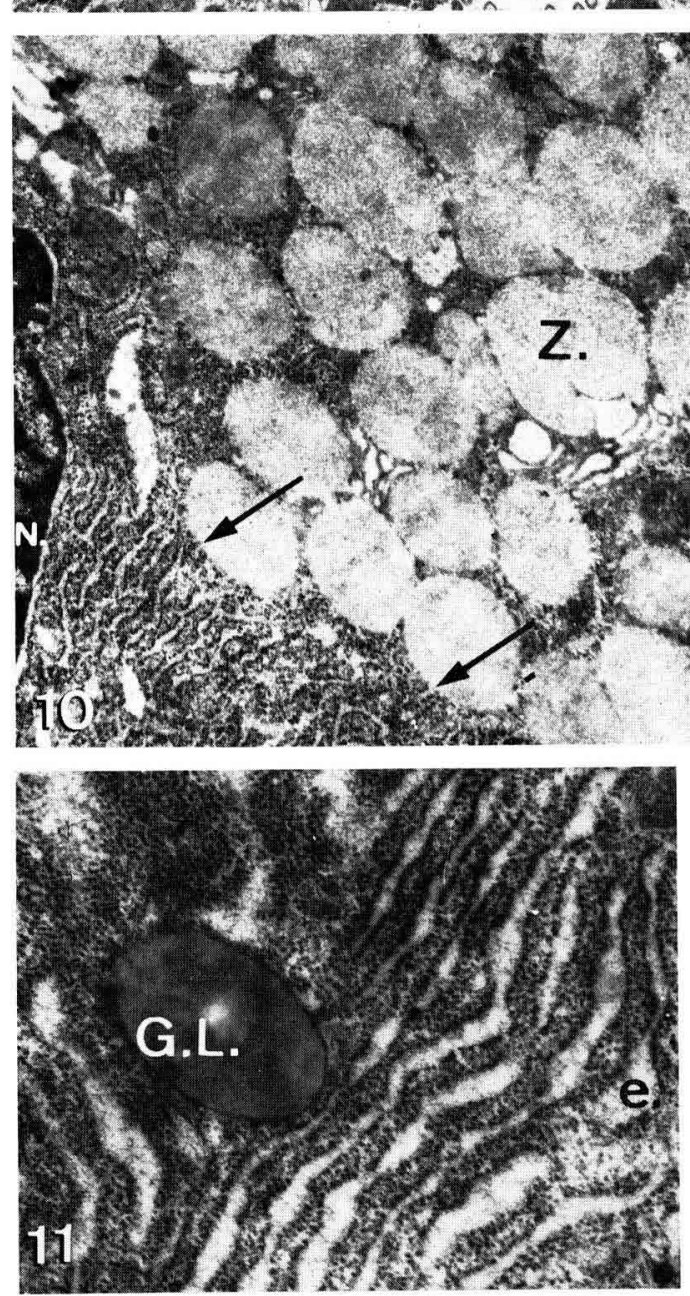




\title{
PLANCHE IV
}

\author{
FIG. 13.
}

Epithélium stomacal chez un animal témoin. Les grains de mucus apicaux, très denses sont serrés les uns contre les autres. $\times 1500$.

FIG. 14.

Epithélium stomacal prélevé dans une poche isolée. Les grains de mucus sont moins serrés que dans la figure précédente. La taille des cellules est semblable à celle des animaux témoins. $\times 1500$.

FIG. 15.

Les grains de mucus (M.) des cellules superficielles occupent la partie supranucléaire et notamment la plus apicale. Les cellules voisines sont reliées entre elles par des desmosomes (d.) nombreux. L. S. : lumière stomacale. $\times 12000$. 
Ann. Biol. anim. Bioch. Biophys., 1971, 11 (1).

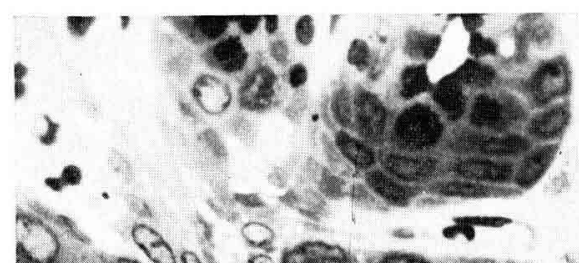

2. $=01000$
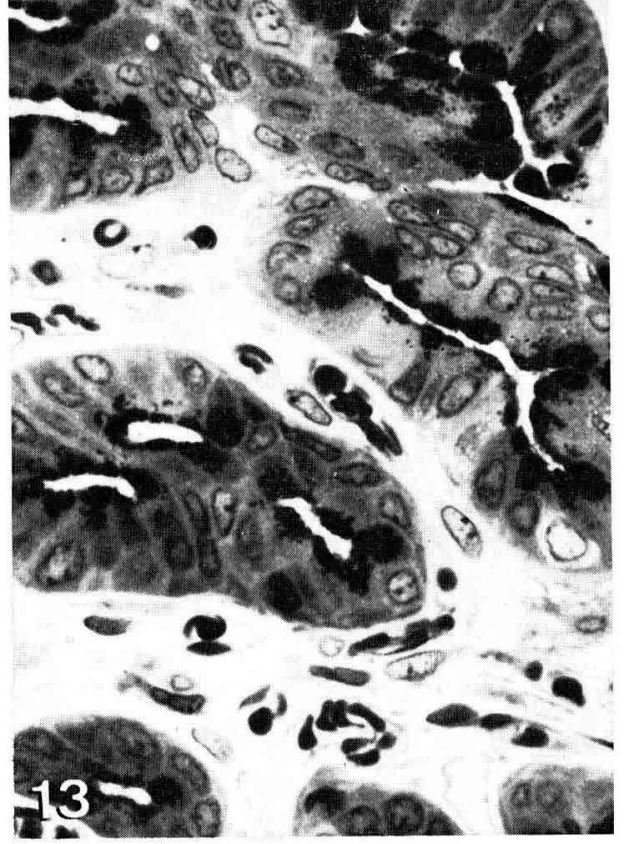

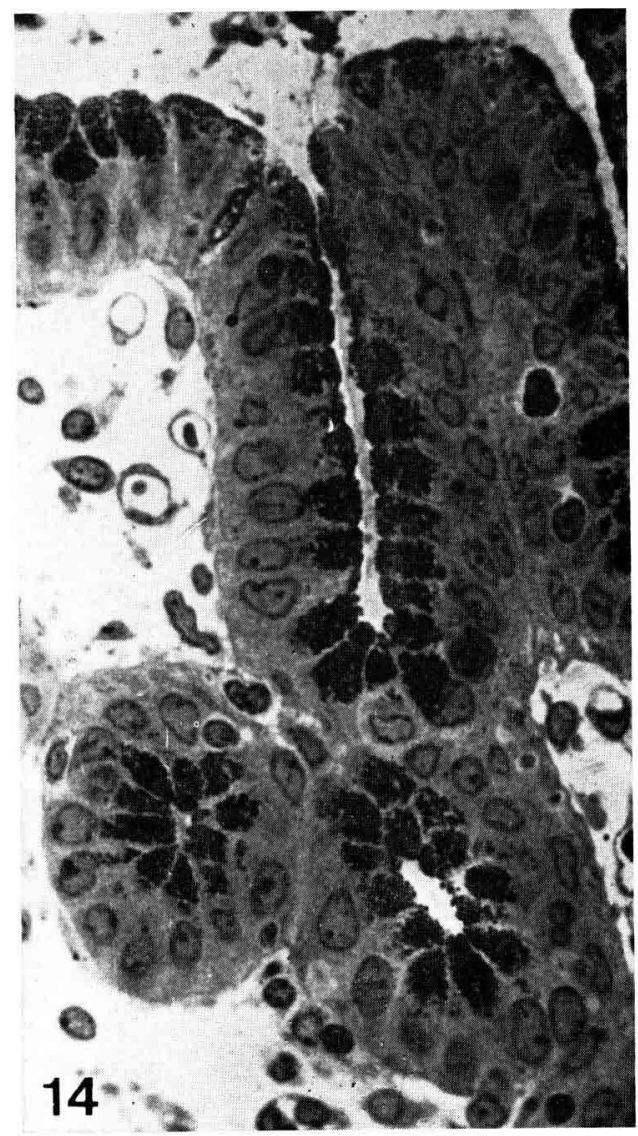

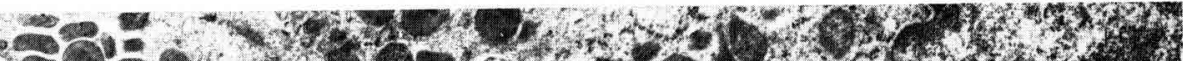

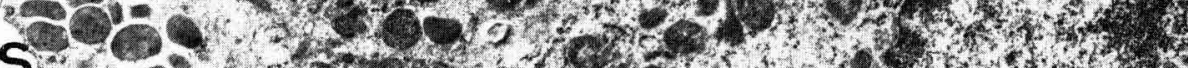
L.S.2 - "

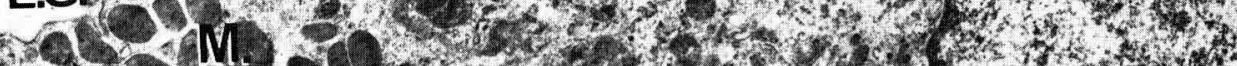

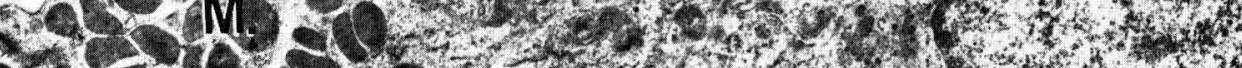

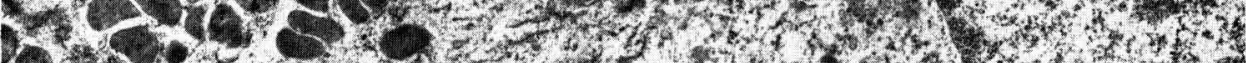

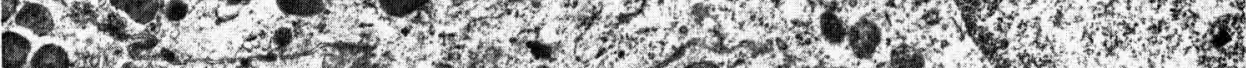

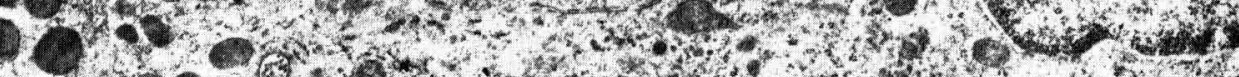

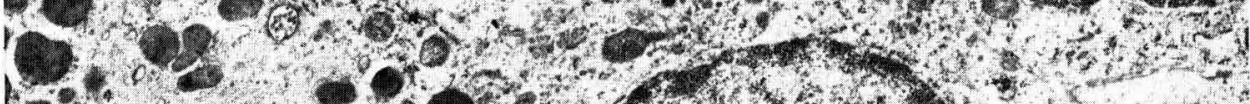

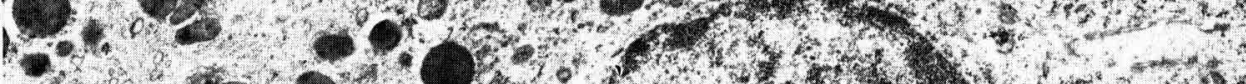

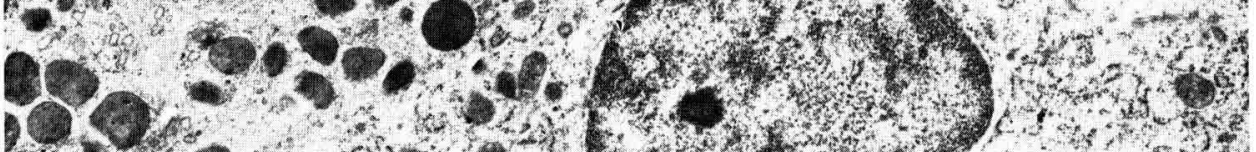

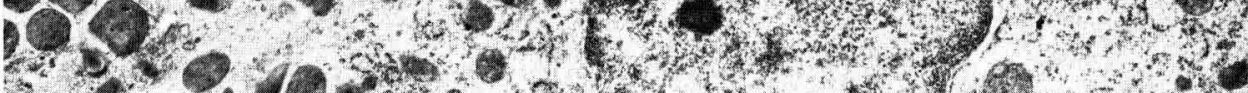

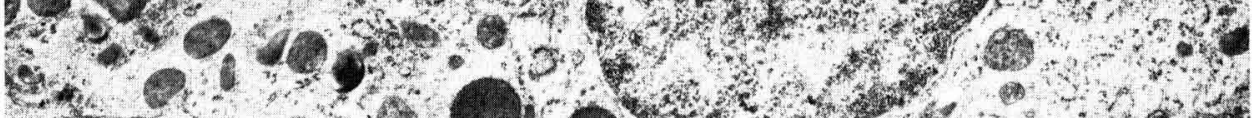
1 a d.

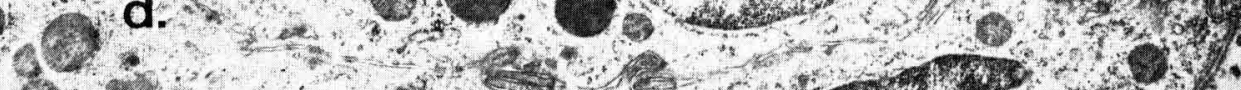

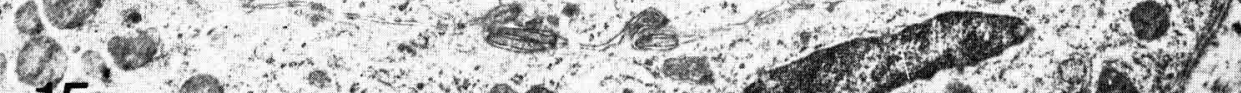
15) 0 - .

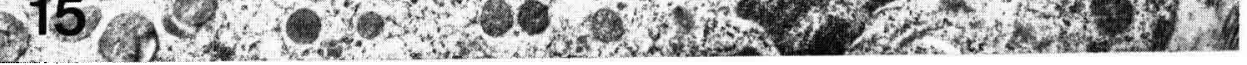




\section{PLANCHE V}

Fig. 16.

Les corps multivésiculaires (c. m.) sont circonscrits par une membrane tristratifiée et ils contiennent des microvésicules à contenu dense. $\times 25000$.

FIG. 17.

Lysosome (Ly.). $\times 15000$.

FIG. 18.

Appareil de Golgi (Go.) avec dans son voisinage des grains de mucus en voie de formation (G. f.). Mitochondrie (m.). On voit bien sur ce cliché l'abondance de ribosomes libres. $\times 15000$.

FIG. 19.

Cellule muqueuse du collet : grains de mucus clairs (M.), appareil de Golgi (Go.) sous forme de vésicules et de saccules. L. G. : lumière glandulaire. $\times 15000$.

FIG. 20.

Cellule argyrophile : obsərver que la forme, la densité et la taille des grains de sécrétion sont très variables. Noter la présence de l'inclusion filamenteuse intranucléaire (flèche). $\times 35000$. 


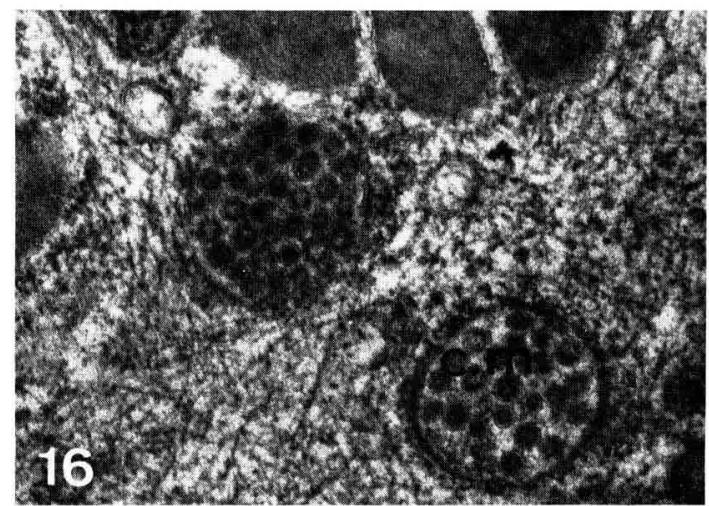

5.6.

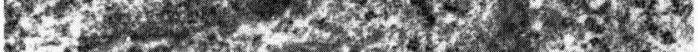

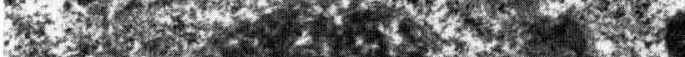

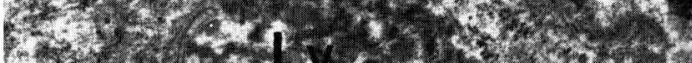

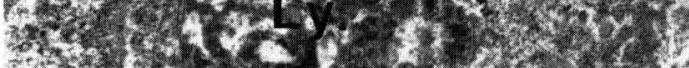

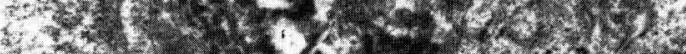

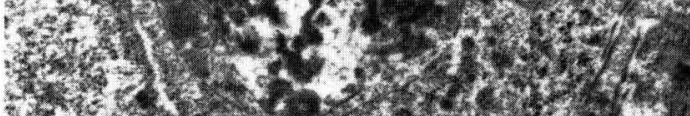

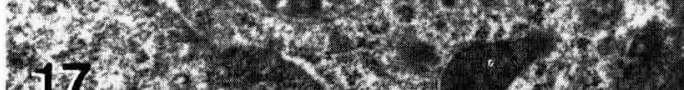

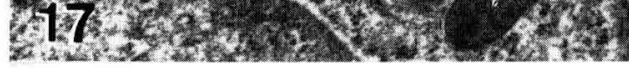

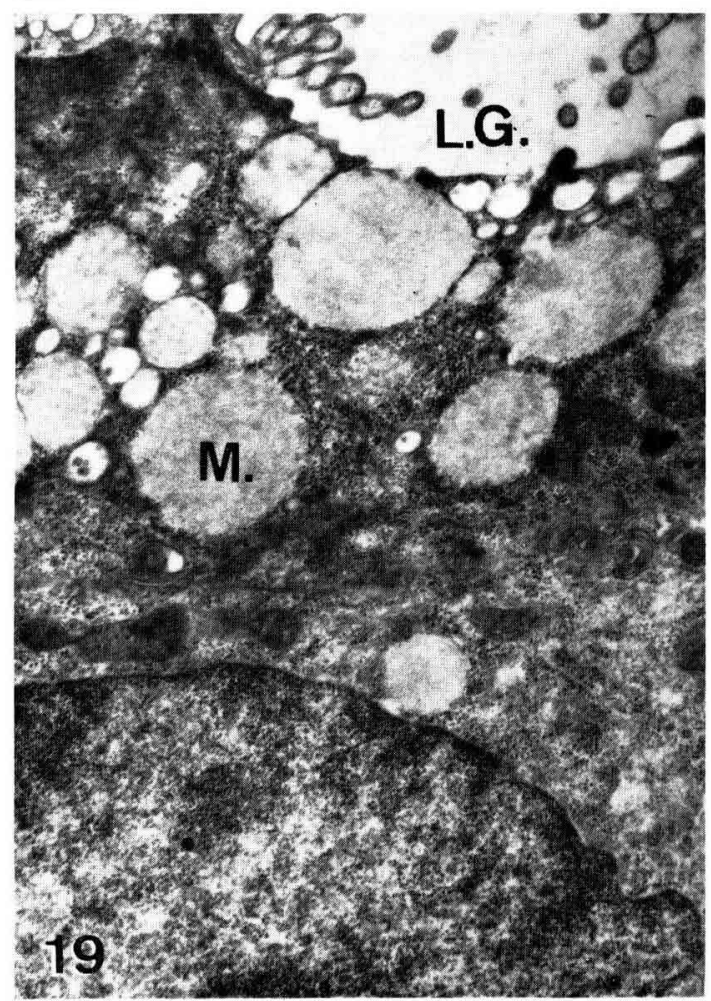

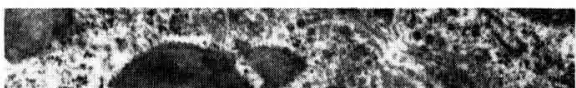

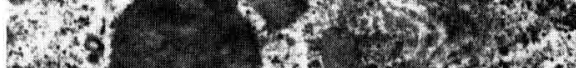

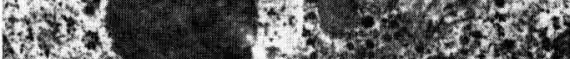

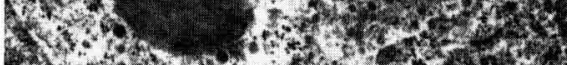
onsts

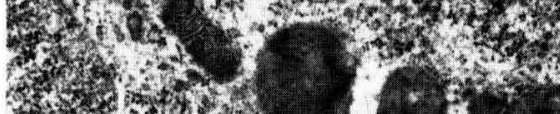

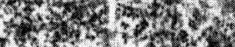

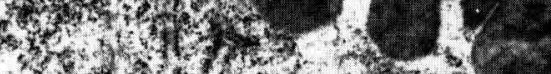
and

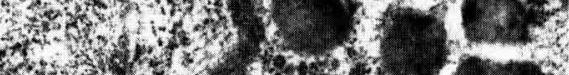

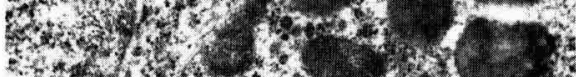

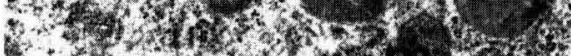

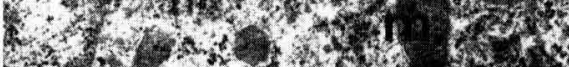
3y

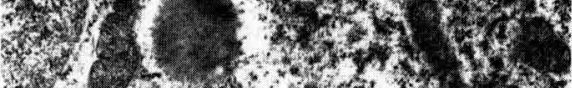

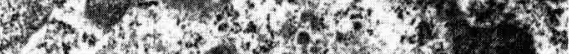
(v)

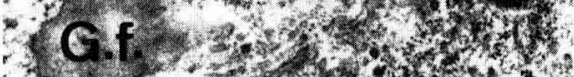
10 $18.60 \% 20$

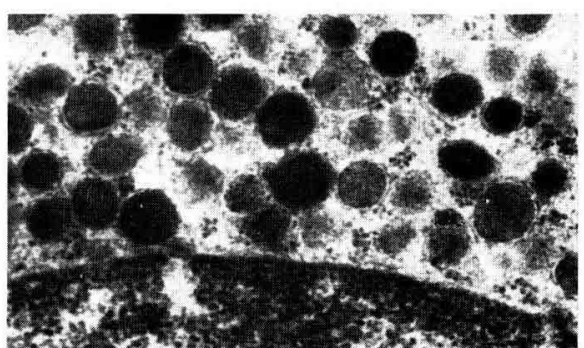

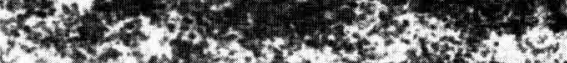

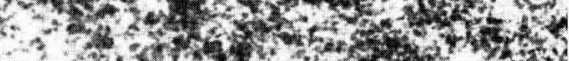

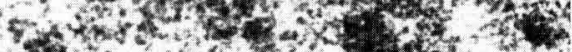
1.

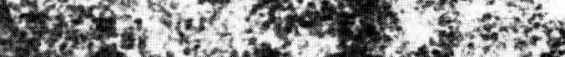
30 .

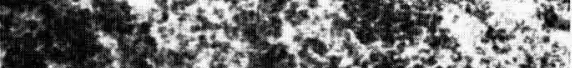

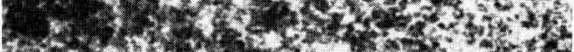

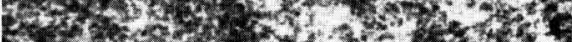

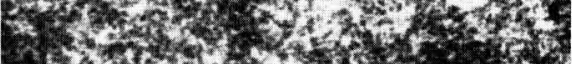

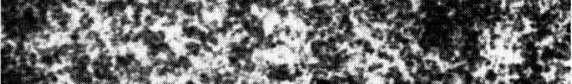

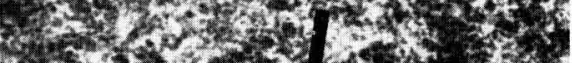

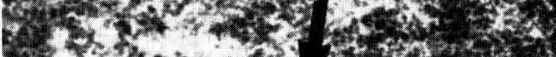
$25 x^{2}=45$

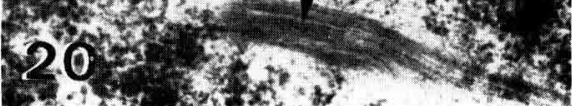

